\title{
Article \\ A Portable Non-Contact Tremor Vibration Measurement and Classification Apparatus
}

\author{
Mohd Zarhamdy Md Zain ${ }^{1}\left(\mathbb{D}\right.$, Ali Zolfagharian ${ }^{2, *(\mathbb{D})}$, Moslem Mohammadi ${ }^{2}\left(\mathbb{C}\right.$, Mahdi Bodaghi $^{3}{ }^{(0)}$, \\ Abd Rahim Abu Bakar ${ }^{1}$ and Abbas Z. Kouzani ${ }^{2}$ (D) \\ 1 Department of System Dynamics \& Control, Faculty of Mechanical Engineering, Universiti Teknologi \\ Malaysia, Skudai 81310, Johor, Malaysia; zarhamdy@mail.fkm.utm.my (M.Z.M.Z.); \\ arahim@utm.my (A.R.A.B.) \\ 2 School of Engineering, Deakin University, Geelong, VIC 3216, Australia; \\ mohammadim@deakin.edu.au (M.M.); abbas.kouzani@deakin.edu.au (A.Z.K.) \\ 3 Department of Engineering, School of Science and Technology, Nottingham Trent University, \\ Nottingham NG11 8NS, UK; mahdi.bodaghi@ntu.ac.uk \\ * Correspondence: a.zolfagharian@deakin.edu.au
}

Citation: Md Zain, M.Z.;

Zolfagharian, A.; Mohammadi, M.; Bodaghi, M.; Abu Bakar, A.R.; Kouzani, A.Z. A Portable Non-Contact Tremor Vibration Measurement and Classification Apparatus. Actuators 2022, 11, 26. https://doi.org/10.3390/ act11010026

Academic Editors: Duk Shin and Matteo Cianchetti

Received: 2 November 2021

Accepted: 12 January 2022

Published: 17 January 2022

Publisher's Note: MDPI stays neutral with regard to jurisdictional claims in published maps and institutional affiliations.

Copyright: (C) 2022 by the authors. Licensee MDPI, Basel, Switzerland. This article is an open access article distributed under the terms and conditions of the Creative Commons Attribution (CC BY) license (https:// creativecommons.org/licenses/by/ $4.0 /)$.

\begin{abstract}
Tremors are the most common type of movement disorder and affect the lives of those experiencing them. The efficacy of tremor therapies varies according to the aetiology of the tremor and its correct diagnosis. This study develops a portable measurement device capable of noncontact measurement of the tremor, which could assist in tremor diagnosis and classification. The performance of this device was assessed through a validation process using a shaker at a controlled frequency to measure human tremors, and the device was able to measure vibrations of $50 \mathrm{~Hz}$ accurately, which is more than twice the frequency of tremors produced by humans. Then, the device is tested to measure the tremors for two different activation conditions: rest and postural, for both hand and leg. The measured non-contact tremor vibration data successfully led to tremor classification in the subjects already diagnosed using a contact accelerometer.
\end{abstract}

Keywords: portable; tremor; movement disorder; non-contact; vibration; measurement

\section{Introduction}

Movement disorder is classified as a neurologic syndrome, and can manifest in two distinct forms: hyperkinetic movement or hypokinetic movement [1,2]. Hyperkinetic movement is characterized by the presence of excessive movement that is not voluntarily performed and is uncontrollable [3]. Individuals who have this sort of movement disorder may notice that when some parts of their body move inadvertently, they are unable to control the movement, such as their hands shaking while carrying a cup of water. Hypokinetic movement, on the other hand, is known as the difficulty in initiating movement and a reduction in the ability to perform a voluntary movement in terms of speed and amplitude [4]. Most Parkinson's disease patients usually experience hypokinetic movement, which is noticeable in their walk as the steps become shorter and slower [5].

Tremor is the most often seen movement condition in clinical practice, and it is a kind of hyperkinetic movement disorder [6]. By definition, tremor is the involuntary movement of body parts or limbs in a rhythmic and oscillating manner [7]. The aetiology of tremors could originate from various causes, including fatigue, drug intake, and neurologic disorders [8,9]. The aetiology of the tremor will have an effect on how the tremor behaves, such as how the tremor is activated, which body parts are affected, and how strong and how often the tremor is. Each aetiology has its own set of tremor result characteristics. For instance, Parkinson's disease patients usually experience tremors of 4 to $6 \mathrm{~Hz}$, activated in a resting condition; on the other hand, essential tremor patients will experience tremors of 4 to $11 \mathrm{~Hz}$, activated in a postural condition [10]. 
Basically, a tremor has two main activation conditions, namely rest and action [6-8,11]. Rest is the condition in which the affected body part is fully supported against gravity, such as when the patient places their hand on top of a table in a relaxed manner. Action is the condition where the tremor is activated during any manoeuvre of the body part that voluntarily contracts the muscle. This can be divided into five other conditions: postural, kinetic, intention, isometric, and task-specific.

There are various types of treatments possible for tremor patients, and in some cases, it is possible to completely cure or suppress the tremor [12]. However, each aetiology of tremor only responds to a specific type of treatment or stimulant [13]. The diagnosis of tremor patients must be done correctly and quickly so that the patient can get the treatment they need to improve their quality of life. Usually, the diagnosis of a tremor patient is made by considering four criteria: the phenomenology of the tremor, frequency and amplitude, familial history, and neurological examination [14]. Among these four criteria, only the frequency and amplitude require some measurement to get the exact value produced by the tremor. Currently, clinicians are measuring this criterion subjectively by palpation and observation. Based on the consensus statement made by the Movement Disorder Society in 1998 on tremors, they determined that tremors for each aetiology have their specific range of frequency and activation condition [15]. Therefore, the ability to accurately measure tremor frequency will considerably enhance the diagnosis of tremor patients. Among a few sensors suitable to measure tremors, the most common one used is the accelerometer due to its practicality [12,16]. Additionally, there have been studies using laser displacement sensors as the transducer to develop a system to measure tremors [17], introducing a different approach from using an accelerometer, as it uses a contactless measurement method and is capable of measuring the tremor displacement.

This study is conducted to develop a measurement approach capable of measuring the tremor in both the hand and the leg. Adding the system's capability to measure the tremor in the leg allows clinicians to measure tremors for a wider group of tremor aetiologies, as specific aetiologies only affect the hand, the leg, or both. This study aims to design a measurement device capable of fulfilling the following targets:

(i) To measure tremors occurring in the hand and leg.

(ii) To adapt for measuring both rest and postural tremors.

(iii) To impose minimal interference through non-contact and portable measurement.

\section{Methodology}

\subsection{Measurement Rig Design Specifications}

This measurement device is designed based on the specifications of the sensor and measuring mode flexibility while ensuring portability. The sensor selected to be used with this device is a laser displacement sensor. With the use of this sensor, it is possible to measure the movement of the body parts affected by tremors without having the sensor in contact with the body parts. This is due to the laser displacement sensor characteristics, which measure displacement based on the reflective ray of the laser projected by the sensor [18-20].

As for the measuring mode, the device is designed to have five different measuring modes, which cover two different measuring conditions for both the hand and the leg. Four of the measuring modes are for measuring tremors in the hand, while the last one is for measuring tremors in the leg. To enhance the portability of this device, the structure of the device is made in such a way that it is detachable into smaller parts for ease of transportation and is easy to assemble. Furthermore, this device is also designed with the flexibility of adapting to various human sizes and measuring locations. To ensure this feature, the position of the sensor is designed to be easily adjusted to ensure that the distance of the sensor from the surface of the body parts is maintained at about half of the sensor range. 


\subsection{Fabrication}

Components fabricated for this device can be divided into five separate groups: frames, sensor holder, hinges and bases, measurement accessories, and external hardware.

\subsubsection{Frames}

The rig is split into several small components to increase portability, as this allows the rig to pack and deploy more compactly. The connections between the parts are made with custom-designed fittings to enable the rig to be disassembled and assembled with ease. The bases are the bottom part of the rig, which is responsible for stabilizing the rig by preventing it from toppling and wiggling during the measurement. The bases are made of a combination of 3D-printed parts and acrylic plates. The 3D-printed parts were designed to serve as sockets to ease attachment and detachment of the bases from the aluminium beam. The frames act as the main structure for this device. Figure 1 shows the fully assembled frame of the device together with the sensor. The frames are made up of $25 \mathrm{~mm}$ by $25 \mathrm{~mm}$ squared hollow aluminium rods. To form a complete structure, the device requires three aluminium rods with a length of $100 \mathrm{~mm}$.

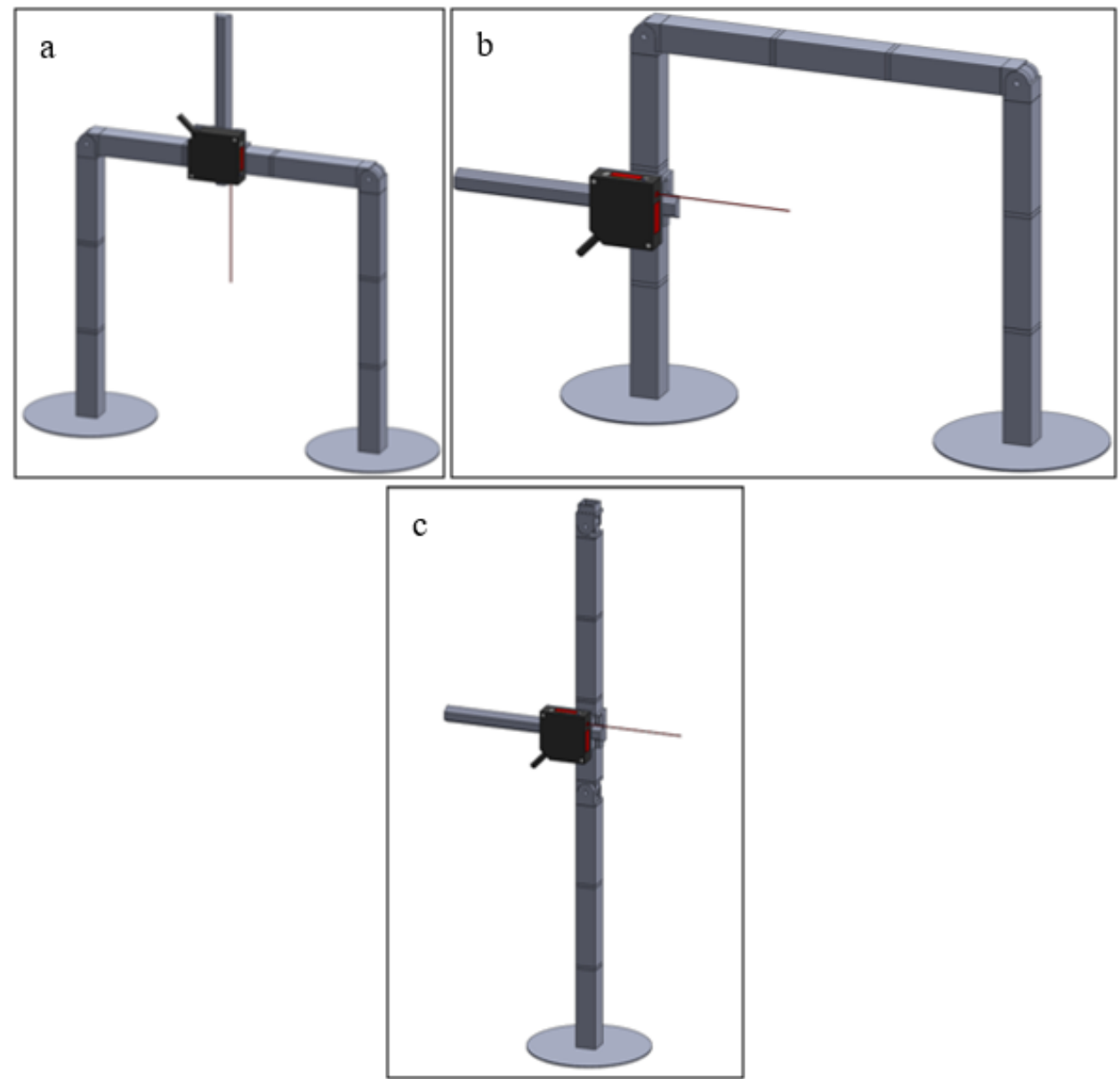

Figure 1. 3D modelling of the rig: in hand measurement setup, (a) up-down measurement, (b) left-right measurement, and (c) in the leg measurement setup.

\subsubsection{Laser Displacement Sensor and Sensor Holder}

A laser displacement sensor is used to measure the tremor activity of the patient's body parts. The laser displacement sensor used is the HL-G112-A-C5 (Panasonic, Ōsaka, Japan) sensor with a measuring range of $120 \pm 60 \mathrm{~mm}$. The detailed specifications of the laser displacement sensor are shown in Table 1. 
Table 1. Detail specifications of selected laser displacement sensor.

\begin{tabular}{cc}
\hline Specification & HL-G112-A-C5 \\
\hline Measuring Range & $120 \pm 60 \mathrm{~mm}$ \\
Resolution & $8 \mu \mathrm{m}$ \\
Sampling Frequency & $500 \mathrm{~Hz}, 1 \mathrm{kHz}, 2 \mathrm{kHz}, 5 \mathrm{kHz}$ \\
Supply Power & $24 \mathrm{VDC}$ \\
(Voltage $\backslash$ Current) & less than $100 \mathrm{~mA}$ \\
Output Voltage & 0 to $10.5 \mathrm{~V}$ \\
\hline
\end{tabular}

The sensor holder is responsible for integrating a laser displacement sensor into the frame. Figure 2 shows the fabricated sensor holder mounted with the sensor. The sensor holder also allows the sensor position to be adjusted perpendicular to the frame it is mounted to.

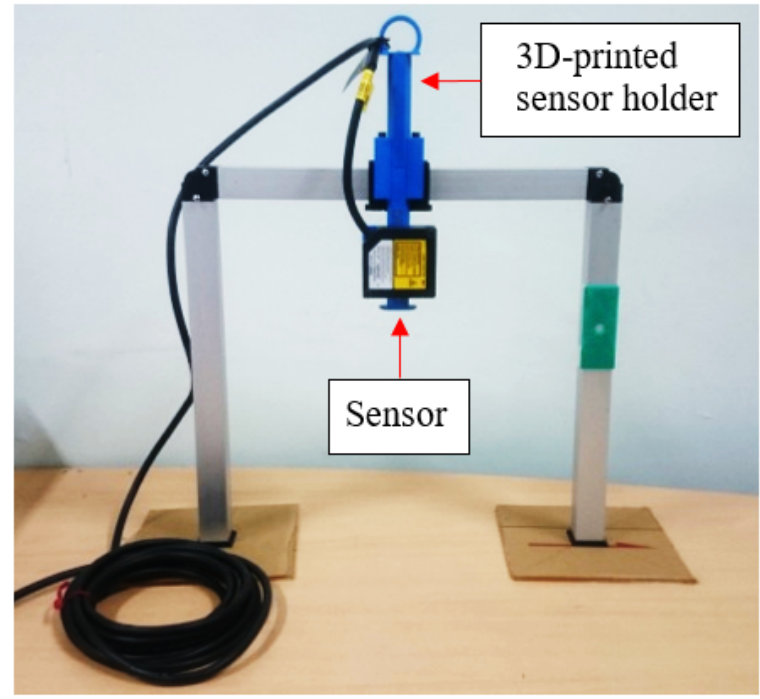

Figure 2. Fully assembled structure together with the sensor holder mounted with the sensor.

\subsubsection{Hinges and Bases}

All three aluminium frames are assembled using the hinges as the medium. The hinges are designed in a manner that allows the aluminium frame and the hinge to be joined by simply plugging the frame into the hinge. The hinges can revolve at an angle of 90 degrees, allowing the measurement device to transform from hand-measuring mode to leg-measuring mode. The bases for the measuring devices are also plugged in with the frames to ensure the stability of the device during measurement. The structure is easily assembled by plugging it together, and this allows the device to be transported easily in the form of small parts, which also means a quick setup.

\subsubsection{Measurement Accessories}

This device also has three accessories that are utilized to assist in the measuring process. The accessories are the $120 \mathrm{~mm}$ jig, measuring plates, and pointing target, which can be seen in Figure 3. The $120 \mathrm{~mm}$ jig serves the purpose of assisting in estimating the midpoint of the distance between the sensor's sensing range. As for the measuring plates, they provide a flat surface for the sensor to obtain a measurement, which improves the accuracy of the measurement made by the sensor. 


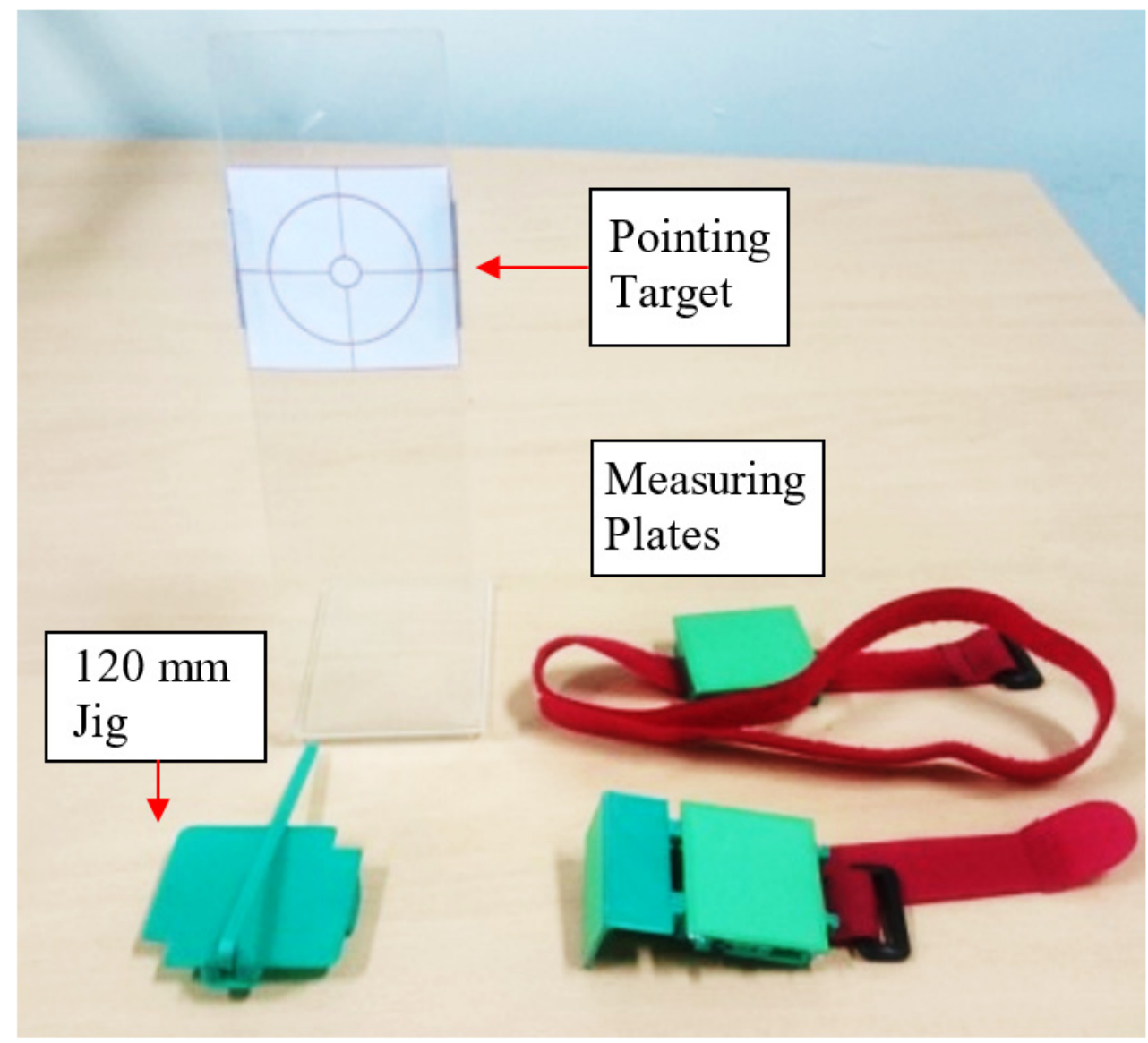

Figure 3. Accessories used to assist measuring process.

During the measurement of hand tremor for postural condition, the pointing target is used together with the hand's measuring plate to assist the user in maintaining the hand's position in postural measurement and ensure that the hand stays within the laser displacement measuring range. The pointing target comprises a transparent acrylic plate bent into an " $\mathrm{L}$ " shape and an adjustable target sheet. The target sheet is slid into position in such a way that the centre of the sheet target will position the hand in the middle of the laser-displacement sensor's measuring range.

\subsubsection{External Hardware and Data Acquisition Unit}

This measurement device requires a set of external hardware, which includes a DC power supply unit, a data acquisition module, and a computer, as shown in Figure 4 . The DC power supply is used to provide $24 \mathrm{~V}$ to the operating sensor, while the DAQ module extracts the raw measuring data from the sensor. The data acquisition unit used is the USB-4716, a portable data acquisition module manufactured by Advantech. With the use of Universal Serial Bus (USB) as the means of connection, this data acquisition could be connected to most computers, as most computers nowadays come with a USB port. This data acquisition unit had a sampling rate of 200 thousand samples per second. Based on the raw data extracted, a computer processes the data through a program that converts the data into the frequency domain. 


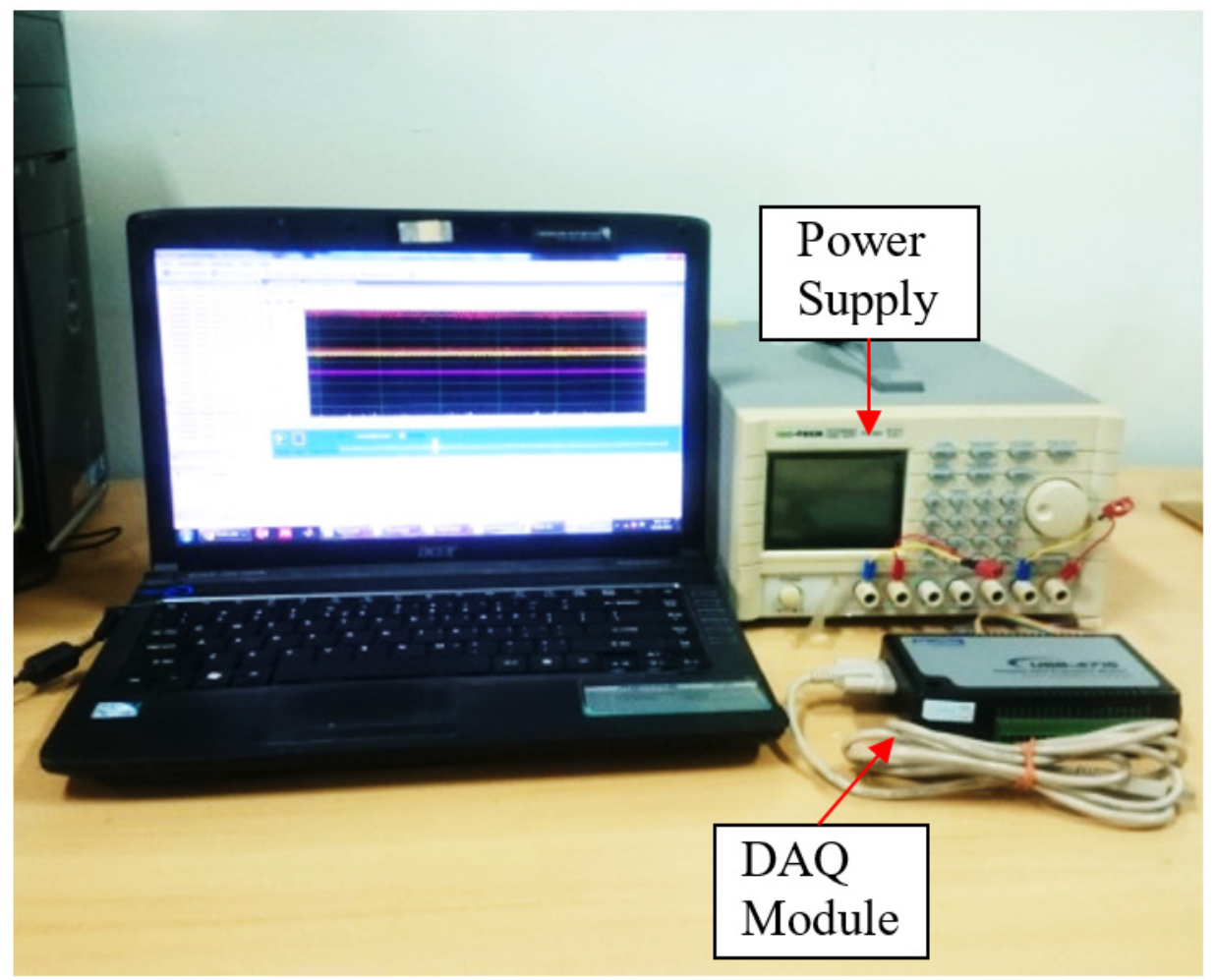

Figure 4. A set of required external hardware.

\subsection{Classification Algorithm}

The tremor classification system consists of two main parts, acquisition and classification, as in Figure 5. The acquisition part of the program is responsible for extracting and processing data obtained from the laser displacement sensor. Both parts were constructed by using MATLAB (R2017a, The MathWorks Inc., Natick, MA, USA) as a base. Here, the data undergo a filtering and transformation process. The raw data are first filtered to cancel out the noise using a low pass filter with a limit value of $20 \mathrm{~Hz}$ based on the characteristic of human tremors, which lies in the range of 4 to $18 \mathrm{~Hz}$. The filtered data then undergo a second order of differentiation before being transformed from the time domain to the frequency domain through Fast Fourier Transform to obtain the condition of the data with respect to the frequency. The value of the frequency mode that had the highest magnitude is passed to the classifier for further handling.

The classification portion of the program is conducted through a scoring process that determines the location of the dominant frequency with respect to the range of frequencies for each tremor group. If a frequency lies in the middle of a specific tremor group's frequency range, it gives that group a higher score compared to the other tremor groups. Therefore, the group that possesses a higher score is more likely to be the associated tremor group. Table 2 shows the tremor frequency range for the tremor group, which is defined by the 1998 Consensus Statement of the Movement Disorder Society [16]. 


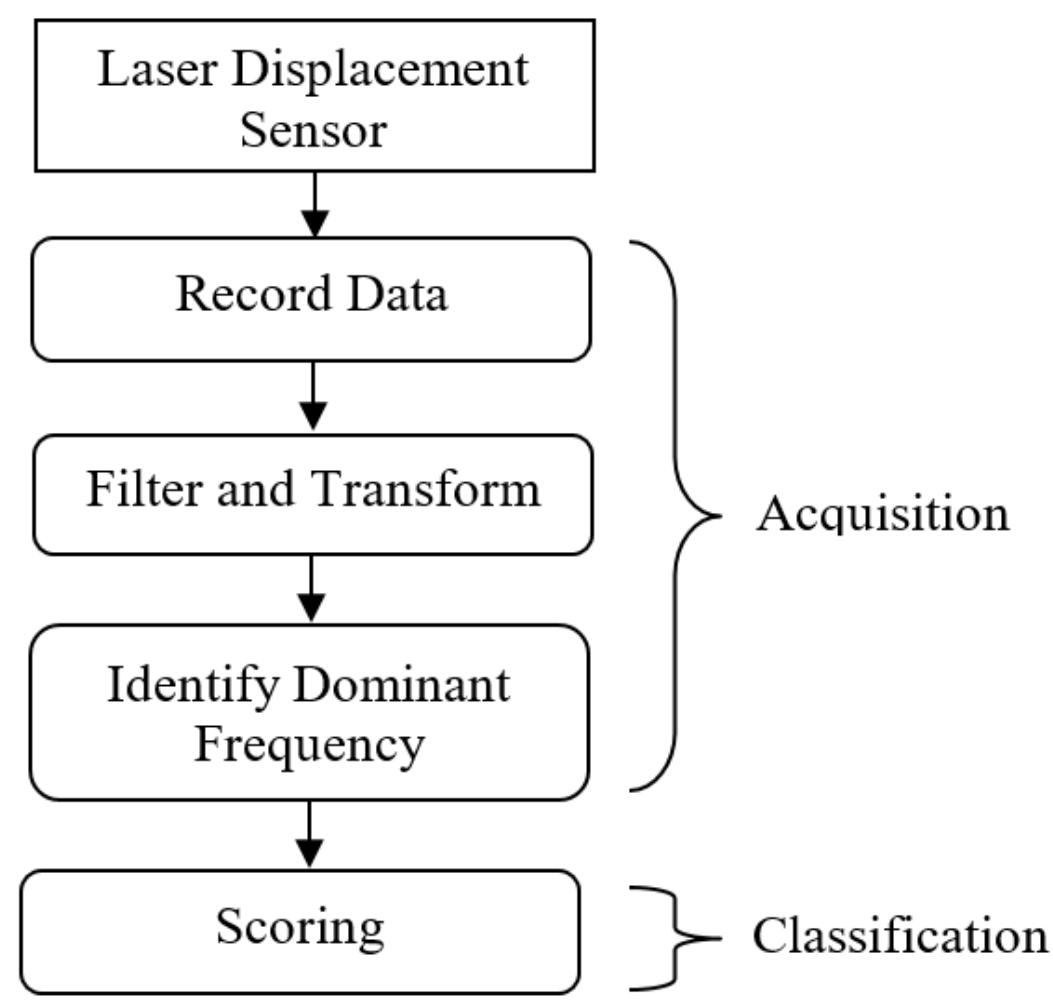

Figure 5. Flow chart of the classification system.

Table 2. Typical activation condition and frequency range for tremors, defined in the 1998 Consensus Statement of the Movement Disorder Society $*^{* *}, *$ two-star posture is the necessary condition for diagnosis, but tremors related to the diagnosis may occur in one-star situations) [16].

\begin{tabular}{|c|c|c|}
\hline Diagnosis & Frequency Range (Hz) & Situation that Tremor Occurs \\
\hline Enhanced physiologic tremor & $5-11.2$ & $\begin{array}{l}\text { ** Posture } \\
* \text { Goal-directed motion }\end{array}$ \\
\hline \multicolumn{3}{|l|}{ Essential tremor syndromes } \\
\hline Classical essential tremor & $4.3-11$ & $\begin{array}{c}\text { ** Posture } \\
{ }^{*} \text { Goal-directed motion, rest }\end{array}$ \\
\hline Primary orthostatic tremor & $12.7-17.8$ & $\begin{array}{c}* * \text { Posture } \\
* \text { Goal-directed motion }\end{array}$ \\
\hline Task and position specific tremor & $5.6-11.3$ & $\begin{array}{l}* * \\
* \text { Goal-directed motion, posture }\end{array}$ \\
\hline \multicolumn{3}{|l|}{ Unclassified tremor } \\
\hline Dystonic tremor & $4.4-10.1$ & $\begin{array}{c}\text { ** Posture, goal-directed motion } \\
\text { * Rest }\end{array}$ \\
\hline Parkinsonian tremor & 3.7-6.6 (rarely happen in 6.6-10.9 too) & $\begin{array}{l}\qquad * * \text { Rest } \\
* \text { Goal-directed motion, posture }\end{array}$ \\
\hline Cerebellar tremor & 2.1-4.4 (rarely happen in $4.4-7.1$ too) & $\begin{array}{c}* * \text { Posture, goal-directed motion } \\
* \text { _ }\end{array}$ \\
\hline Holmes tremor & $1.3-5$ & $\begin{array}{l}* * \text { Goal-directed motion, rest } \\
\text { * Posture }\end{array}$ \\
\hline Palatal tremor & $0.6-3.5$ & $\begin{array}{c}* * \text { Rest } \\
*-\end{array}$ \\
\hline Neuropathic tremor & $5-11.3$ & $\begin{array}{c}\text { ** Posture } \\
* \text { Goal-directed motion }\end{array}$ \\
\hline Toxic and drug induced tremor & $3.1-11.4$ & ${ }^{*}$ Goal-directed motion, rest, posture \\
\hline Psychogenic tremor & $3.9-10.8$ & ${ }^{*}$ Goal-directed motion, rest, posture \\
\hline
\end{tabular}


The processes of producing score for each of dominant frequency obtained were determined by using the Formula (1):

$$
\text { Score }=\left[1-\left(\frac{|(\operatorname{Max} R-D F)-(D F-\operatorname{Min} R)|}{R}\right)\right] \times 100
$$

where $D F, R, \operatorname{Max} R$, and $\operatorname{Min} R$, are dominant, tremor group's range, minimum, and maximum frequencies, respectively.

Equation (1) is used in determining the score obtained for each group with respect to each tremor group frequency characteristic. Any dominant frequency that lies perfectly in the centre of a tremor group's frequency range will result in the tremor group's obtaining a hundred points for the score. However, if the dominant frequency of the tremor measured lies outside of the tremor group frequency, the product of subtraction for the dominant frequency with either the upper or lower limit of the tremor group frequency results in a negative value. Therefore, the program will avoid calculating the score for tremor groups whose dominant frequency does not lie in between these limits.

\section{Results and Validations}

The performance of this device was assessed through a validation process. The validation was conducted to verify whether the device can record the vibration produced by a shaker induced at a known frequency for both hand and leg tremors. This validation process was conducted by having the device record the movement created by a mini shaker with a $3 \mathrm{~mm}$ stroke length. The mini shaker was set to move at a frequency of $50 \mathrm{~Hz}$, which is twice the frequency produced by a human tremor. Figure 6 illustrates the setup used for the validation process. The recorded data were analysed by converting them into the frequency domain with the Fast Fourier Transform, and the most dominant peak of the signal was identified. The calculated mean for dominant frequencies obtained from three sets of readings is $49.4 \mathrm{~Hz}$, which is $1.22 \%$ less than the $50 \mathrm{~Hz}$ induced by the mini shaker.

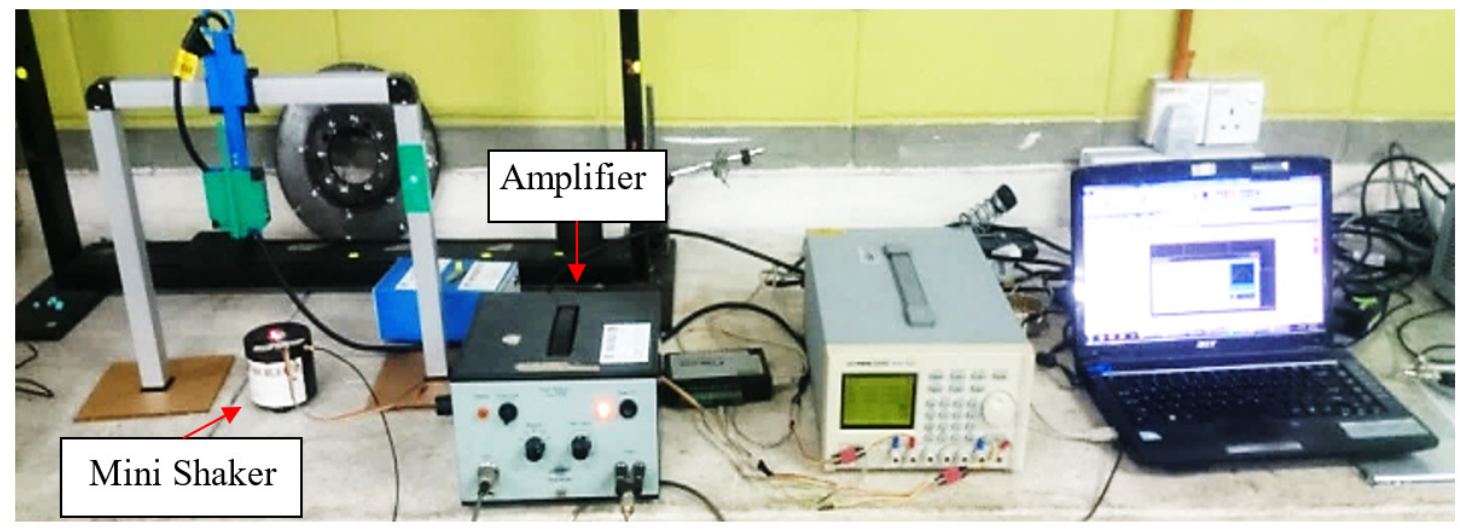

Figure 6. Validation process setup by recording the vibration of a mini shaker.

In this research, the subject's hand and leg tremors were recorded in postural and resting positions. In measuring the tremor in the postural position, the limbs are required to use the muscles to maintain their position. As a result, to measure the hand, the subject had to sit properly on a chair and stretch their arm forward perpendicular to the body, as shown in Figure 7a. The subject needed to simply stand up straight to measure the leg, as shown in Figure 7c. As for the resting position, the muscles of the limb being measured were required to relax. As a result, for the measurement of the hand, the hand was placed on a table while the subject was sitting, as shown in Figure $7 \mathrm{~b}$. The leg was measured with the subject sitting firmly on a chair with their feet on the floor, as shown in Figure 7d. The measurement task was conducted within $10 \mathrm{~s}$ for postural and another 2 min for rest positions, while the measurement set-up preparation and calibration took almost 2 min for each measurement. 

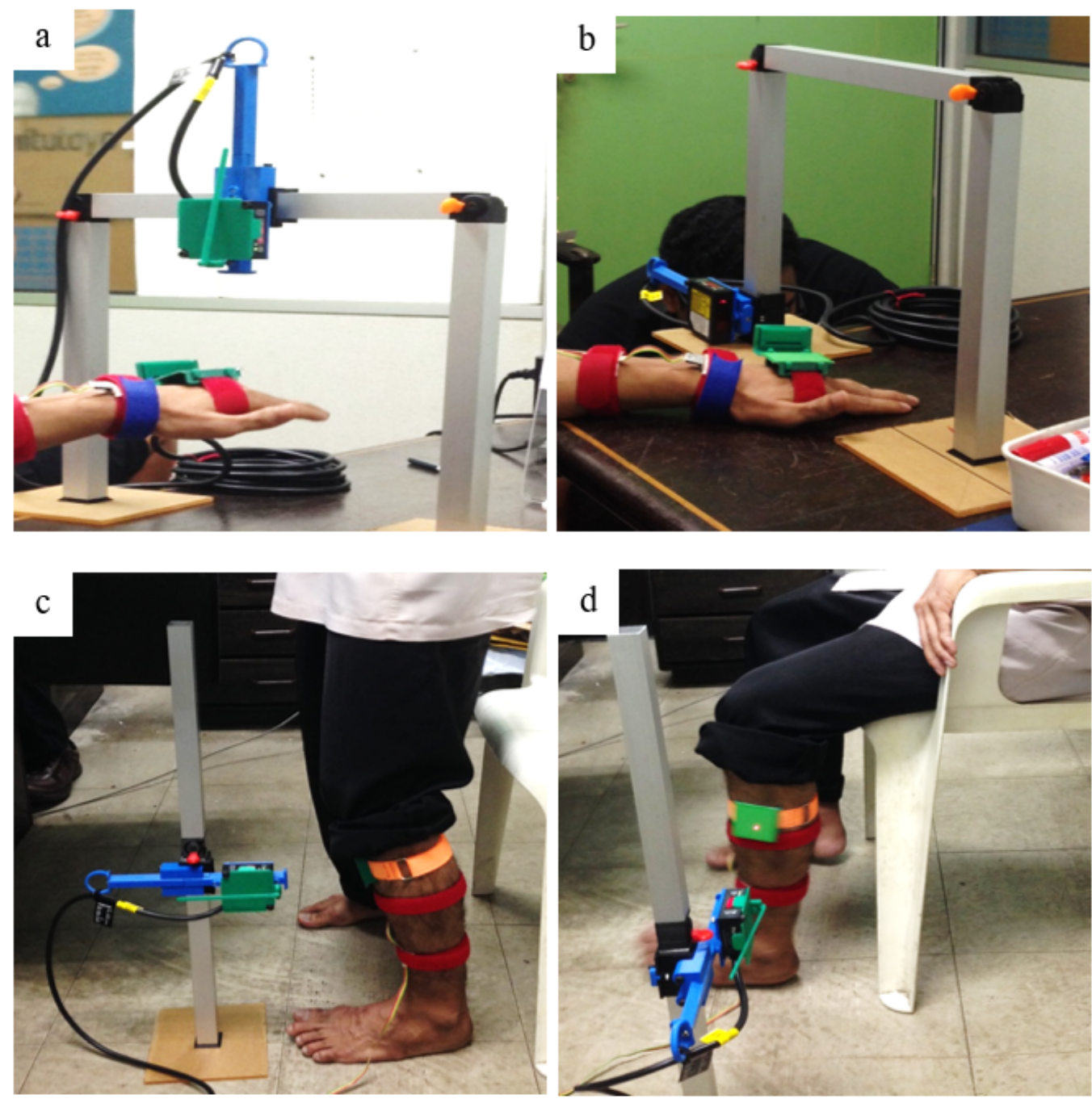

Figure 7. Measurement of hand and leg (a) hand in posture situation, (b) hand in the rest situation, (c) leg in standing situation, (d) leg in rest situation.

The accelerometer used as a secondary sensor was the ADXL330, which is capable of measuring acceleration in three different axes. A data logger was used in this for the purpose of extracting and recording the data from the DAQ unit. The data logger used in this study was software provided by Advantech, which is the manufacturer of the DAQ unit used for this study. Since the programming software used, MATLAB, is unable to directly communicate with the DAQ unit to obtain the required data, the data logger acted as an intermediary by storing the data in a format readable by MATLAB. The data logger was set to record the data at a rate of $2 \mathrm{kHz}$ and store the data in Comma-Separated Value (.csv) format.

The conditioning step is handled in the program constructed using MATLAB. In this step, three different processes are conducted on the data recorded. The processes were filtration, differentiation, and Fast Fourier Transform. The filtration process was performed using a low-pass Butterworth filter with a cut-off frequency of $20 \mathrm{~Hz}$, which filters out noises at a frequency higher than the human tremor frequency.

As for differentiation, the data obtained from the laser displacement sensor were in the form of displacement and caused the tremor frequency to be less dominant due to the swaying motion of the body parts. This swaying motion caused the magnitude of the frequency below $1 \mathrm{~Hz}$ to appear dominant. Since there are tremor groups with low-frequency tremors, it is not suitable to use a high-pass filter in this situation, and this is where the differentiation process comes into play. Through differentiation, the movement 
caused by sway motion will have less effect on the data as the sway motion will have a lower rate of movement over time, and second-order differentiation is applied in this process. The filter selected to be used on the data conditioning was the Butterworth filter, which was set to filter out noises above the cut-off frequency specified in the program. The cut-off frequency was set to a value of $20 \mathrm{~Hz}$ because the highest value of frequency expected to be generated by a human tremor is below $18 \mathrm{~Hz}$, which lies in the Orthostatic Tremor Group. To improve the ability of the user to handle the program, developed for the tremor group classification, it was constructed using MATLAB's Graphical User Interface module, as shown in Figure 8.

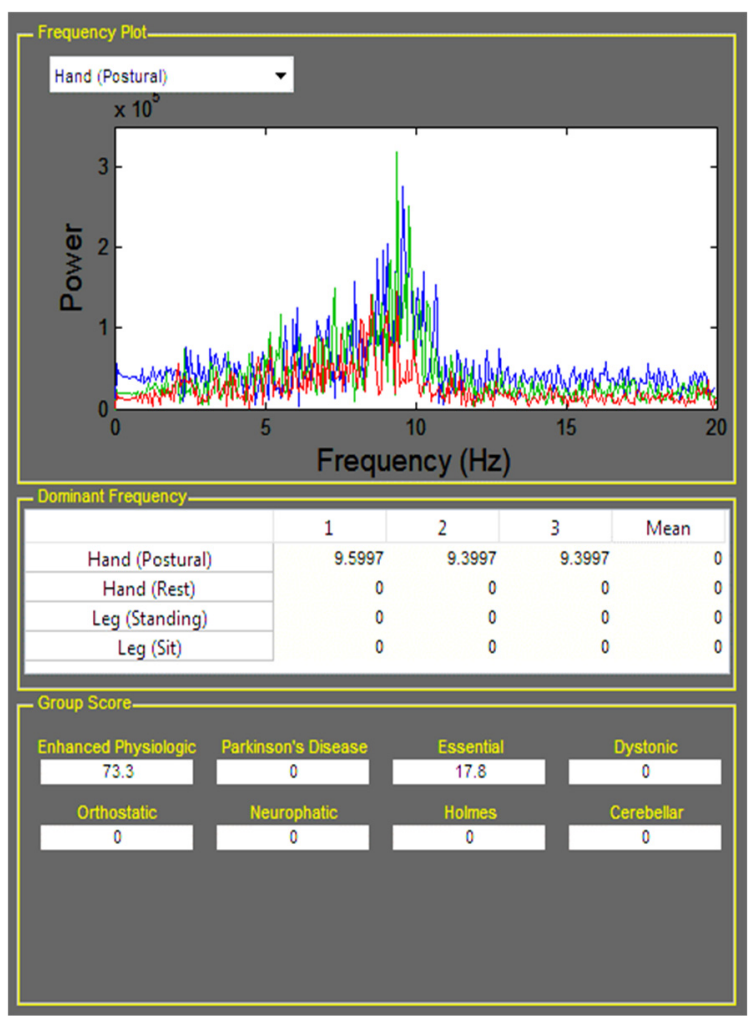

(a)

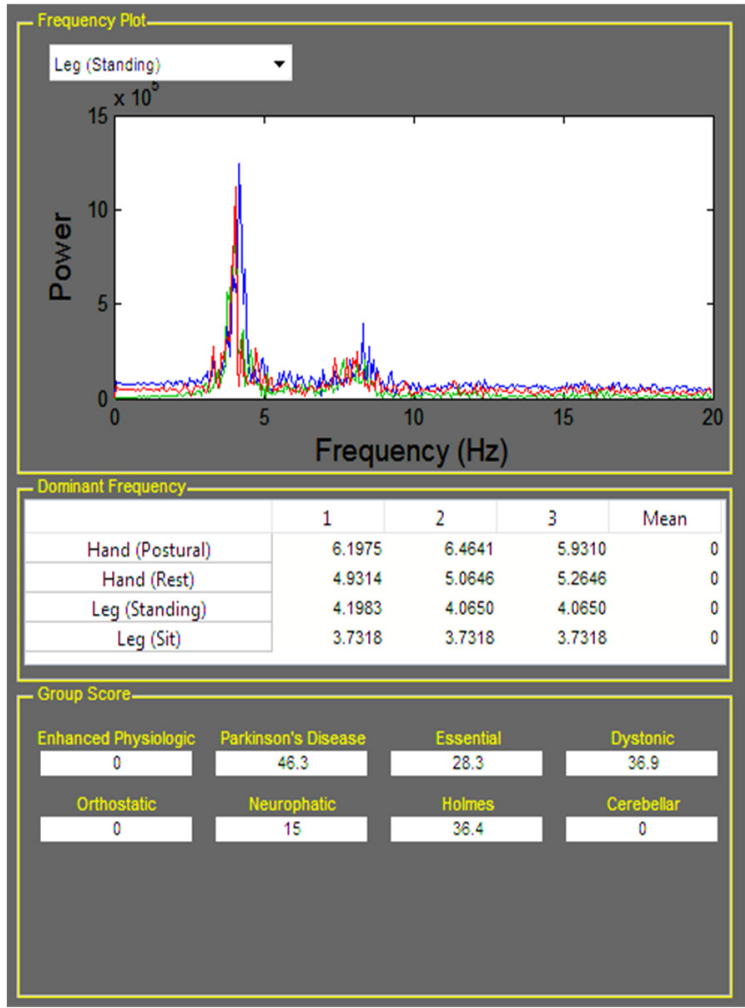

(b)

Figure 8. Program interface classification after analysing and plotting power spectral density of four signals from the measured data in four different situations; (a) based on the scores, enhanced physiologic has been diagnosed; (b) Parkinson's has the highest score, so the algorithm diagnosis is Parkinson's disease.

The sets of data for each subject were analysed by the classification programs constructed and reported in Tables 3 and 4 . Among four of the subjects, two subjects already knew the reason behind their tremor. The subjects are Subject 1 , who was diagnosed with Parkinson's Disease, and Subject 2, who experienced tremors after the muscle suffered from fatigue due to sustaining load for a long time. The remaining subjects were Subject 3 and Subject 4, who did not have any known cause for their tremors. Based on the score received by Subject 1 , the score computed by the program agrees with the one diagnosed by medical personnel, as the score obtained shows that Parkinson's Disease is the highest. The same was also true for Subject 2, who had the highest score for enhanced physiologic tremor as the subject experienced tremors after experiencing muscle fatigue. As for Subject 3 and Subject 4, who do not have any known cause for their tremor problems, the score computed by the program showed that Subject 3 had a high score for Holmes Tremor and Cerebellar Tremor, while Subject 4 had the highest score for Enhanced Physiologic. 
Table 3. List of frequency for each subject.

\begin{tabular}{cccccccccccccc}
\hline \multirow{2}{*}{ Measuring Condition } & \multicolumn{3}{c}{ Subject 1 } & \multicolumn{3}{c}{ Subject 2 } & \multicolumn{3}{c}{ Subject 3 } & \multicolumn{3}{c}{ Subject 4 } \\
\cline { 2 - 11 } & $\mathbf{1}$ & $\mathbf{2}$ & $\mathbf{3}$ & $\mathbf{1}$ & $\mathbf{2}$ & $\mathbf{3}$ & $\mathbf{1}$ & $\mathbf{2}$ & $\mathbf{3}$ & $\mathbf{1}$ & $\mathbf{2}$ & $\mathbf{3}$ \\
\hline Hand Postural & 6.20 & 6.47 & 5.93 & 9.60 & 9.40 & 9.40 & 2.60 & 3.13 & 3.13 & 9.92 & 9.86 & 9.66 \\
Hand Rest & 4.93 & 5.07 & 5.26 & - & - & - & - & - & - & - & - & - & - \\
Leg Postural & 4.20 & 4.07 & 4.07 & - & - & - & 3.33 & 3.53 & 3.00 & - & - & - & - \\
Leg Rest & 3.73 & 3.73 & 3.73 & - & - & - & - & - & - & - \\
\hline
\end{tabular}

Table 4. List score for each tremor group.

\begin{tabular}{ccccc}
\hline Tremor Group & Subject 1 & Subject 2 & Subject 3 & Subject 4 \\
\hline Enhanced Physiologic Tremor & 0 & 73.3 & 0 & 90.9 \\
Parkinson Disease & 46.3 & 0 & 12.6 & 0 \\
Essential Tremor & 28.3 & 17.8 & 0 & 6.1 \\
Dystonic Tremor & 36.9 & 0 & 0 & 0 \\
Orthostatic Tremor & 0 & 0 & 0 & 0 \\
Neurophatic Tremor & 15 & 0 & 0 & 0 \\
Holmes Tremor & 36.4 & 0 & 74.1 & 0 \\
Cerebellar Tremor & 0 & 0 & 74.4 & 0 \\
\hline
\end{tabular}

\section{Discussion}

In this study, we proposed a tremor-measuring device with a contactless sensor to eliminate any extra load on patients' limbs during data recording. The device was designed to be used in clinics to record data from the hand and leg in various situations, including resting and posture conditions. Furthermore, the frame is lightweight, and the hinges are designed to be easy to disassemble and reassemble to make it easier for general practitioners to set the device for different subjects in different situations. Additionally, the structure and the materials used in assisting accessories were chosen to be lightweight tools to minimize their impacts on subjects.

The data measured using the secondary accelerometer, which was placed on the top of the patient's hand, also led to the same classification of the tremor in Subject 1 [21] as the validation of the proposed portable device. The data were collected and calibrated for the resting and postural tremor along with the $\mathrm{Z}$ axis for Subject 1 . However, a different algorithm was used for the quantification of tremor oscillation severity [21]. Based on the results computed, the score obtained by the subjects with a known cause of tremor agrees with their causes as the associated tremor group had the highest score. Furthermore, the program computed the score given to each subject by considering multiple number sets and measurement conditions. Therefore, it can be said that the approach used to obtain the score could assist medical professionals in identifying the cause of the tremor experienced by their patients. The approach proposed by this research provides information regarding the tremor and the possibility of any underlying causes.

Studies have shown that adding weight to a tremulous limb could affect tremor characteristics; therefore, the recorded data obtained by attaching accelerometers could be different from the genuine tremor signal of the subject [22]. When compared to previous work on tremor diagnosis strategies, this work demonstrated greater adaptability to various rest and postural tremors in non-contact devices while accurately classifying and ranking tremor severities in both rest and postural tremors. In the portable tremor diagnosis research thus far, heavy devices were attached to the subject's hands and were used to acquire tremor data $[23,24]$.

Previous research used a contact-less sensor to record tremors in the hand [17], but subjects had to hold their hand in a narrow cylinder-shaped tool that made it uncomfortable for patients. Additionally, the mentioned device is not suitable for recording leg tremors. Similar to the current study, Laser Doppler Vibrometer (LDV) and Kinect devices were 
used to obtain tremor signals, with up to a $25 \%$ difference reported in frequencies detected by different devices [25]. However, there was little experimental work on a vibrant device with a specific frequency, and the device's accuracy on portable devices was not reported. Another study to quantify tremors was carried out in 2019 using Kinect 2, and the results showed high precision in detecting tremors with an amplitude of more than $2 \mathrm{~mm}$ (detect frequency with $25 \%$ error) [26]. This device and algorithm could be used for severe tremors with high amplitude in flexion-extension tremors, but in moderate tremors with low amplitude, the device could lead to wrong diagnoses. Tremor quantifications using noncontact capacitive (NCC) sensors showed promising results compared to gyroscope sensors. However, their proposed apparatus is designed for the upper limb in a rest position, and it must be redesigned for the leg and to record an active tremor [27]. Additionally, a study was conducted to record tremors with video processing technology on videos taken by smartphones. Results showed a -0.38 to $+0.35 \mathrm{~Hz}$ difference from results recorded by an accelerometer [28]. These data, however, are only for hand rest and postural position, and there is a need for additional customization for leg tremor classifications.

The proposed signal acquisition system in this paper is designed to have a nearzero effect on subjects' limbs compared to contact measurement devices [21]. Therefore, the recorded data is a better reference to analyse and report the results to specialists. Additionally, it is a general-purpose apparatus fabricated to collect data from any tremulous part of the subject in various positions (rest or active). Furthermore, the device's portability is an advantage that allows specialists to customize the sensor setup for patients.

\section{Conclusions}

The fabricated measurement device was designed for the purpose of accurately recording the movement produced by a human tremor. The device was not only able to record the tremor movement for the frequency parameter, but it was also able to record the amplitude of the tremor in terms of displacement. In validating the capability of the device in measuring the tremor frequency, it was used to measure a vibrating motion at a frequency of $50 \mathrm{~Hz}$. The device was able to produce a mean dominant frequency reading of $49.4 \mathrm{~Hz}$, which is only about a $1.22 \%$ deviation. Therefore, it is possible to use the device to record the tremor level of humans. To further prove the capability of the device to measure human tremors, a study on the performance of the device in measuring actual human tremors will be conducted. As a future improvement, the measuring rig should be designed to automatically adjust the sensor position. This improvement will reduce the time taken by the user to adjust the position of the sensor.

Author Contributions: Conceptualization, M.Z.M.Z. and A.R.A.B.; Formal analysis, A.Z., M.B. and A.R.A.B.; Funding acquisition, M.Z.M.Z. and A.Z.; Investigation, A.Z. and M.M.; Methodology, A.Z., M.B., A.R.A.B. and A.Z.K.; Project administration, M.Z.M.Z., A.Z. and A.R.A.B.; Supervision, A.Z.; Validation, M.M.; Writing—original draft, M.Z.M.Z., M.M. and A.R.A.B.; Writing—review \& editing, A.Z., M.B. and A.Z.K. All authors equally contributed in this manuscript. All authors have read and agreed to the published version of the manuscript.

Funding: This research was funded by Universiti Teknologi Malaysia (UTM) and Faculty of Science, Engineering and Built Environment for 2021 Mini ARC Analog Program (MAAP)—Discovery 253101, Deakin University for financial support.

Informed Consent Statement: Informed consent was obtained from all subjects involved in the study.

Data Availability Statement: The data presented in this study are available on request from the corresponding author.

Acknowledgments: The authors would like to thank Universiti Teknologi Malaysia (UTM) and Deakin University, Australia, for providing the funding and facilities to conduct this research.

Conflicts of Interest: The authors declare no conflict of interest. 
Ethics Approval: All experimental protocols were conducted at University Technology Malaysia. Informed consent was obtained from all participants.

\section{References}

1. Fahn, S. Classification of movement disorders. Mov. Disord. 2011, 26, 947-957. [CrossRef]

2. Fernandez, H.H.; Rodriguez, R.L.; Skidmore, F.M.; Okun, M.S. A Practical Approach To Movement: Disorders Diagnosis and Surgical and Medical Management; Demos Medical Publishing: New York, NY, USA, 2007.

3. Albanese, A.; Jankovic, J. Distinguishing Clinical Features of Hyperkinetic Disorders. In Hyperkinetic Movement Disorders; Wiley: Hoboken, NJ, USA, 2012; pp. 1-14.

4. DeLong, M.R. Primate models of movement disorders of basal ganglia origin. Trends Neurosci. 1990, 13, 281-285. [CrossRef]

5. Bartels, A.L.; Leenders, K.L. Parkinson's disease: The syndrome, the pathogenesis and pathophysiology. Cortex 2009, 45, 915-921. [CrossRef]

6. Bhidayasiri, R. Differential diagnosis of common tremor syndromes. Postgrad. Med. J. 2005, 81, 756-762. [CrossRef]

7. Buijink, A.W.; Contarino, M.F.; Koelman, J.H.; Speelman, J.D.; Van Rootselaar, A.-F. How to Tackle Tremor-Systematic Review of the Literature and Diagnostic Work-Up. Front. Neurol. 2012, 3, 146. [CrossRef]

8. Bain, P.G. The management of tremor. J. Neurol. Neurosurg. Psychiatry 2002, 72, i3-i9.

9. Soad, M.H.; Zain, M.Z.; Hussein, M. Aetiological classification of tremor. In Proceedings of the International Conference on Innovation in Science and Technology (IICIST 2015), Kuala Lumpur, Malaysia, 20 April 2015.

10. Charles, P.D.; Esper, G.J.; Davis, T.L.; Maciunas, R.J.; Robertson, D. Classification of tremor and update on treatment. Am. Fam. Phys. 1999, 59, 1565-15572.

11. Puschmann, A.; Wszolek, Z.K. Diagnosis and Treatment of Common Forms of Tremor. Semin. Neurol. 2011, 31, 65-77. [CrossRef] [PubMed]

12. Grimaldi, G.; Manto, M. Tremor: From Pathogenesis to Treatment. Synth. Lect. Biomed. Eng. 2008, 3, 1-212. [CrossRef]

13. Bötzel, K.; Tronnier, V.; Gasser, T. The Differential Diagnosis and Treatment of Tremor. Dtsch. Ärzteblatt Int. 2014, 111, 225-236. [CrossRef]

14. Kumar, H.; Jog, M. A patient with tremor, part 1: Making the diagnosis. Can. Med Assoc. J. 2011, 183, 1507-1510. [CrossRef]

15. Raethjen, J.; Lindemann, M.; Krack, P. The pathophysiology of tremor. Muscle Nerve 2001, 24, 716-735.

16. Elble, R.; Deuschl, G. Milestones in tremor research. Mov. Disord. 2011, 26, 1096-1105. [CrossRef]

17. As'arry, A.; Cheraghizanjani, A.; Mohd, M.Z.; Hussein, M.; Yusop, Z.M. Development of Measurement Device and Classification for Tremor Patients Behaviour. In Proceedings of the 13th WSEAS International Conference on Mathematical Methods, Computational Techniques and Intelligent Systems, Iasi, Romania, 11-13 May 2006; pp. 23-26.

18. Clark, R. Triangulation Displacement Sensor. 2003. Available online: https://patents.google.com/patent/US6624899B1/en (accessed on 28 October 2021).

19. Khosravani, M.R.; Zolfagharian, A. Fracture and load-carrying capacity of 3D-printed cracked components. Extreme Mech. Lett. 2020, 37, 100692. [CrossRef]

20. Zolfagharian, A.; Kaynak, A.; Bodaghi, M.; Kouzani, A.Z.; Gharaie, S.; Nahavandi, S. Control-Based 4D Printing: Adaptive 4D-Printed Systems. Appl. Sci. 2020, 10, 3020. [CrossRef]

21. Musab, R.; As'Arry, A.; Rezali, K.A.M.; Jalil, N.A.A.; Ahmad, R.M.K.R.; Zain, M.Z.M. Tremor Quantification and its Measurements Using Shimmer. J. Physics: Conf. Ser. 2019, 1262, 012024. [CrossRef]

22. Bazgir, O.; Habibi, S.A.H.; Palma, L.; Pierleoni, P.; Nafees, S. A Classification System for Assessment and Home Monitoring of Tremor in Patients with Parkinson's Disease. J. Med. Signals Sens. 2018, 8, 65-72. Available online: https://www.ncbi.nlm.nih. gov/pmc/articles/PMC5992899/ (accessed on 28 October 2021). [CrossRef]

23. Woods, A.M.; Nowostawski, M.; Franz, E.A.; Purvis, M. Parkinson's disease and essential tremor classification on mobile device. Pervasive Mob. Comput. 2014, 13, 1-12. [CrossRef]

24. Alam, N.; Johnson, B.; Gendreau, J.; Tavakolian, K.; Combs, C.; Fazel-Rezai, R. Tremor quantification of Parkinson's disease-A pilot study. In Proceedings of the 2016 IEEE International Conference on Electro Information Technology (EIT), Grand Forks, ND, USA, 19-21 May 2016; pp. 755-759. [CrossRef]

25. Casacanditella, L.; Cosoli, G.; Ceravolo, M.; Tomasini, E. Non-contact measurement of tremor for the characterisation of Parkinsonian individuals: Comparison between Kinect and Laser Doppler vibrometer. J. Physics: Conf. Ser. 2017, 882, 012002. [CrossRef]

26. Alper, M.A.; Goudreau, J.; Daniel, M. Pose and Optical Flow Fusion (POFF) for accurate tremor detection and quantification. Biocybern. Biomed. Eng. 2020, 40, 468-481. [CrossRef]

27. Oliveira, F.H.M.; Rabelo, A.G.; Luiz, L.M.D.; Pereira, A.; Vieira, M.; Andrade, A. On the Use of Non-Contact Capacitive Sensors for the Assessment of Postural Hand Tremor of Individuals with Parkinson's Disease. In Proceedings of the 2019 41st Annual International Conference of the IEEE Engineering in Medicine and Biology Society (EMBC), Berlin, Germany, 23-27 July 2019; Volume 2019, pp. 6591-6594.

28. Williams, S.; Fang, H.; Relton, S.D.; Wong, D.C.; Alam, T.; Alty, J.E. Accuracy of smartphone video for contactless measurement of hand tremor frequency. Mov. Disord. Clin. Pract. 2021, 8, 69-75. [CrossRef] [PubMed] 